\title{
Quantum computational universality of the Cai-Miyake-Dür-Briegel 2D quantum state from Affleck-Kennedy-Lieb-Tasaki quasichains
}

\author{
Tzu-Chieh Wei, ${ }^{1,2}$ Robert Raussendorf, ${ }^{1}$ and Leong Chuan Kwek ${ }^{3}$ \\ ${ }^{1}$ Department of Physics and Astronomy, University of British Columbia, Vancouver, British Columbia V6T 1Z1, Canada \\ ${ }^{2}$ C. N. Yang Institute for Theoretical Physics, State University of New York at Stony Brook, Stony Brook, NY 11794-3840, USA \\ ${ }^{3}$ Centre for Quantum Technologies, National University of Singapore, 2 Science Drive 3, \\ Singapore and National Institute of Education and Institute of Advanced Studies, \\ Nanyang Technological University, 1 Nanyang Walk, Singapore
}

(Dated: July 16, 2021)

\begin{abstract}
Universal quantum computation can be achieved by simply performing single-qubit measurements on a highly entangled resource state, such as cluster states. Cai, Miyake, Dür, and Briegel recently constructed a ground state of a two-dimensional quantum magnet by combining multiple AffleckKennedy-Lieb-Tasaki quasichains of mixed spin-3/2 and spin-1/2 entities and by mapping pairs of neighboring spin-1/2 particles to individual spin-3/2 particles [Phys. Rev. A 82, 052309 (2010)]. They showed that this state enables universal quantum computation by single-spin measurements. Here, we give an alternative understanding of how this state gives rise to universal measurementbased quantum computation: by local operations, each quasichain can be converted to a 1D cluster state and entangling gates between two neighboring logical qubits can be implemented by single-spin measurements. We further argue that a $2 \mathrm{D}$ cluster state can be distilled from the Cai-Miyake-DürBriegel state.
\end{abstract}

PACS numbers: 03.67.Ac, 03.67.Lx, 75.10.Jm

\section{INTRODUCTION}

Measurement-based quantum computation (MBQC) provides a framework where quantum computation [1] is achieved by single-spin measurements on a highly entangled resource state [2, 3]. The first known resource state is the so-called cluster state on a square lattice [4]. Graph states [5] on other two-dimensional regular lattices were also shown to be universal resources $[6$. It turns out that universal resource states are shown to be rare, of zero measure [7. However, resource states beyond graph states have been constructed [8], mostly due to the understanding of MBQC from the perspective of matrix-product-states (MPS) and projected-entangledpair-states (PEPS) 9, 10. In fact, there exist universal resource states that are unique ground states of twobody interacting Hamiltonians [11 15], even though cluster states are not [16].

Among the small class of known universal states is the construction by Cai, Miyake, Dür, and Briegel (CMDB) 13. It is based on chains of Affleck-KennedyLieb-Tasaki (AKLT) states [17, originally constructed in the setting of condensed matter physics. It was shown earlier that one-dimensional spin-1 AKLT states can serve as resources for restricted quantum computations [8, 18, and universal computation can be achieved by active coupling of many such $1 \mathrm{D}$ chains [18]. The merit of the CMDB construction [13] is to avoid such active coupling by mapping two connecting spin- $1 / 2$ entities into one spin- $3 / 2$ entity, and patching many 1D AKLT quasichains into a two-dimensional structure. Moreover, the gap above the ground state of the constructed 2D model was shown to be finite [13]. Here, we show that by local operations, the ground state of an
AKLT quasichain of mixed spin-3/2 and spin-1/2 entities can be converted to a 1D cluster state. Furthermore, entangling gates between two logical qubits on neighboring quasichains can be implemented by measuring the spin-3/2 particle connecting them. This provides an alternative understanding of the quantum-computational universality using the CMDB state. Moreover, we argue that from this state a $2 \mathrm{D}$ cluster state can be distilled by local operations.

The structure of the remaining of the paper is as follows. In Sec. II we review the 1D AKLT quasichain. In Sec. III we review the 2D Cai-Miyake-Dür-Briegel model. In Sec. [V] we show how the 1D quasichain AKLT state can be locally converted to a 1D cluster state. In Sec. V we show how to understand the quantum computational universality of the CMDB state and how it can be locally converted to a $2 \mathrm{D}$ cluster state. In Sec. VI]we make some concluding remarks.

\section{1D AKLT QUASICHAIN}

In 13 the 1D AKLT model defined on a quasichain is considered, which consists of spin-3/2 particles (labeled by $A_{i}$ 's) located at the backbone and spin- $1 / 2$ particles (labeled by $b_{i}$ 's) connected to $A$ 's, as shown in Fig. 1a. The Hamiltonian of the quasichain is [13]

$$
H=\sum_{i=1}^{N-1} P_{A_{i}, A_{i+1}}^{S=3}+\sum_{i=1}^{N} P_{A_{i}, b_{i}}^{S=2}+P_{A_{1}, b_{0}}^{S=2}+P_{A_{N}, b_{N+1}}^{S=2},
$$

where $P_{u, v}^{S}$ represents the projector onto the spin- $S$ subspace of the joint $u$ and $v$ spins, which can be expressed as a polynomial of $\vec{S}_{u} \cdot \vec{S}_{v}$. The original AKLT chain 
(a)

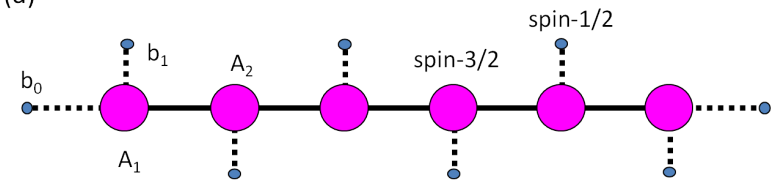

(b)

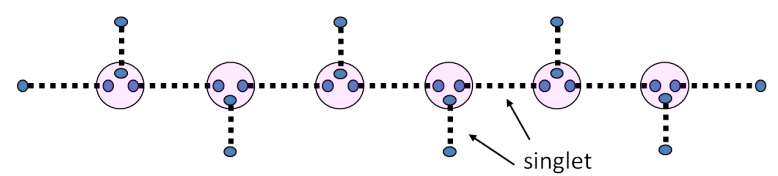

FIG. 1: (Color online) 1D AKLT quasichain. (a) The larger circles represent spin- $3 / 2$ particles and are connected by thick solid lines. They form the backbone of the quasichain. The smaller circles represent spin- $1 / 2$ particles, which are connected via dashed lines to corresponding spin- $3 / 2$ particles. (b) The schematic of the ground state of the 1D AKLT quasichain. Each spin-3/2 can be regarded as three virtual spin$1 / 2$ particles, each forming a singlet with the neighbor virtual qubit. The three qubits at $A$ site is then projected into their symmetric subspace, with the projection indicated by a transparent circle.

is defined on a chain of spin-1 particles, with the ends terminated by spin-1/2 particles [17. The quasichain, similar to the original AKLT chain, can be shown to possess a unique ground state with a finite spectral gap [13. As illustrated in Fig. 1p, the ground state is a valencebond-solid state of the AKLT type, in which every $A$ is composed of three virtual spin-1/2 particles, each forming a singlet pair with its neighboring spin- $1 / 2$ particle, followed by a projection $\Pi_{S}$ (defined below) of the three virtual qubits to their symmetric subspace. To be more precise, the ground state is the following valence-bond state,

$$
\left|\psi_{\mathrm{AKLT}}\right\rangle \sim \underset{A}{\otimes} \Pi_{S, A} \underset{\text { edge } e}{\otimes}|\phi\rangle_{e},
$$

where $|\phi\rangle_{e}=(|01\rangle-|10\rangle) / \sqrt{2}$ is a singlet state for the pair of qubits residing on the (dashed) edge $e$; see Fig. 1 1 b.

The correspondence of the states of the three virtual particles to those of a spin- $3 / 2$ particle is given as follows,

$$
\begin{aligned}
& |000\rangle \leftrightarrow\left|\frac{3}{2}, \frac{3}{2}\right\rangle, \\
& |111\rangle \leftrightarrow\left|\frac{3}{2},-\frac{3}{2}\right\rangle \\
& |W\rangle \equiv \frac{1}{\sqrt{3}}(|001\rangle+|010\rangle+|100\rangle) \leftrightarrow\left|\frac{3}{2}, \frac{1}{2}\right\rangle, \\
& |\bar{W}\rangle \equiv \frac{1}{\sqrt{3}}(|110\rangle+|101\rangle+|011\rangle) \leftrightarrow\left|\frac{3}{2},-\frac{1}{2}\right\rangle,
\end{aligned}
$$

where the qubit states $|0 / 1\rangle$ are the eigenstates of spin$1 / 2$ angular momentum operators $\hat{S}^{2}$ and $\hat{S}_{z}$, i.e., $|0\rangle \equiv$ $|1 / 2,1 / 2\rangle$ and $|1\rangle \equiv|1 / 2,-1 / 2\rangle$. The projector onto

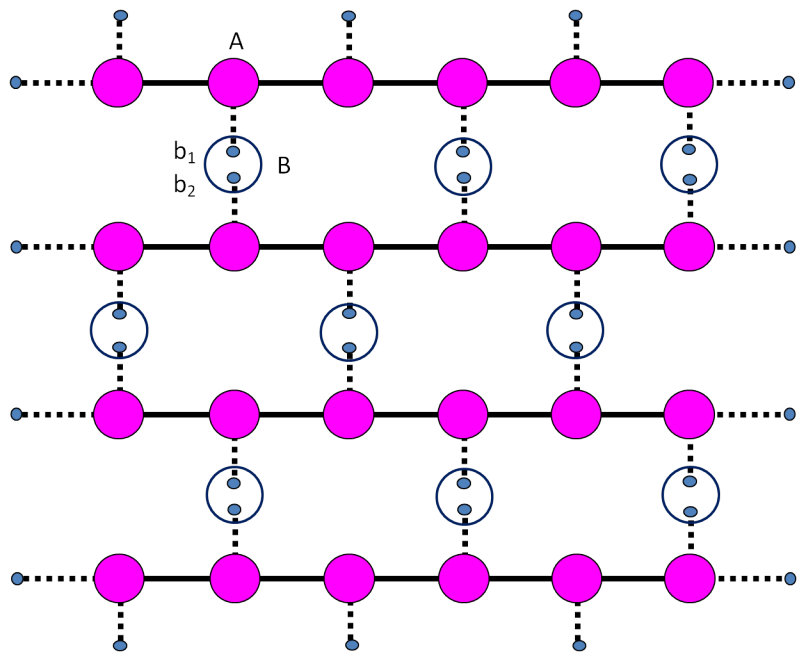

FIG. 2: (Color online) The 2D Cai-Miyake-Dür-Brigel model. The model consists of many AKLT quasichains and merge the two neighboring spin- $1 / 2$ particles, e.g., $b_{1}$ and $b_{2}$, to a single spin-3/2 particle $B$ via $U$ in Eq. (4).

the symmetric subspace can be simply written as $\Pi_{S} \equiv$ $|000\rangle\langle 000|+| W\rangle\langle W|+| \bar{W}\rangle\langle\bar{W}|+| 111\rangle\langle 111|$. The quasichain AKLT state in Eq. (2) is written in terms of the virtual-qubit representation for the central spin-3/2 particles; the relabeling in Eq. (3) provides a translation to the spin- $3 / 2$ representation. We note that the quantization-axis label on the states is implicitly assumed to be along the $z$-axis. In the following, when necessary, we will explicitly write out the quantization axis.

\section{2D CAI-MIYAKE-DÜR-BRIEGEL MODEL}

To build up a 2D model with a finite gap above the ground state, Cai et al. stack up 1D quasichains and their mirror images in a staggered way, as shown in Fig. 2. The two neighboring spin- $1 / 2$ particles, e.g., $b_{1}$ and $b_{2}$, possess in total four levels and can be formally mapped to a spin-3/2 particle $B$ by a unitary transformation $U$,

$$
U=\sum_{m_{1}, m_{2}= \pm 1 / 2}\left|\frac{3}{2}, m_{1}+2 m_{2}\right\rangle_{B}\left\langle\frac{1}{2},\left.m_{1}\right|_{b_{1}}\left\langle\frac{1}{2},\left.m_{2}\right|_{b_{2}} .\right.\right.
$$

The Hamiltonian of the resulting 2D system is obtained from the sum of Hamiltonians of many 1D quasichains (1), followed by the unitary transformations $U$ 's merging all pairs of neighboring $b$ 's to single spin- $3 / 2 B$ 's. For example, a term $f\left(\vec{S}_{A} \cdot \vec{s}_{b_{1}}\right)$ in the Hamiltonian (1) is transformed to $f\left(\vec{S}_{A} \cdot U \vec{s}_{b_{1}} U^{\dagger}\right)$, with $U \vec{s}_{b_{1}} U^{\dagger}$ and similarly $U \vec{s}_{b_{2}} U^{\dagger}$ being observables of the spin-3/2 B. For the explicit form of the resulting 2D Hamiltonian, we refer the readers to the paper by Cai et al. [13. Because of the spectral gap in the quasichains, the whole system by patching them together using the unitary $U$ 's has a gap as well. We note that in the following discussions of quan- 
tum computational universality it is actually convenient to treat $B$ in terms of the two virtual spin- $1 / 2 b$ particles before the unitary $U$. Any unitary transformation on the composite $b_{1}$ and $b_{1}$ is a local unitary transformation on $B$. Moreover, joint measurements on $b_{1}$ and $b_{2}$ is a local measurement on $B$. We also remark that with open boundary conditions the CMDB model contains boundary spin- $1 / 2$ particles. To remove these and make the model consist solely of spin- $3 / 2$ particles, one can use periodic boundary conditions by merging two associated opposite-end spin-1/2's to one spin-3/2.

\section{FROM THE GROUND STATE OF THE AKLT QUASICHAIN TO A 1D CLUSTER STATE}

In this section we consider the ground state of the AKLT quasichain and shall show that it can be locally converted to a $1 \mathrm{D}$ cluster state. The local conversion is achieved by a generalized measurement, also called positive-operator-value measure (POVM) [1] at each site, as was used previously in the 2D AKLT state on the honeycomb lattice [14. The idea is to "project" the four levels of a spin-3/2 particle down to two levels, forming an effective qubit. To bring out the "hidden" cluster state from the quasichain AKLT state, we generalize the projection and introduce the following POVM at each $A$ site, which consists of three rank-two elements 14

$$
\begin{aligned}
& F_{A, z}=\sqrt{\frac{2}{3}}\left(|+3 / 2\rangle\left\langle+3 /\left.2\right|_{z}+\mid-3 / 2\right\rangle\left\langle-3 /\left.2\right|_{z}\right),\right. \\
& F_{A, x}=\sqrt{\frac{2}{3}}\left(|+3 / 2\rangle\left\langle+3 /\left.2\right|_{x}+\mid-3 / 2\right\rangle\left\langle-3 /\left.2\right|_{x}\right),\right. \\
& F_{A, y}=\sqrt{\frac{2}{3}}\left(|+3 / 2\rangle\left\langle+3 /\left.2\right|_{y}+\mid-3 / 2\right\rangle\left\langle-3 /\left.2\right|_{y}\right),\right.
\end{aligned}
$$

where the subscripts $x, y$ and $z$ indicating the measurement outcome in fact represent the effective quantization axes and we have omitted the total spin magnitude $S=3 / 2$ in the Dirac brackets. In contrast to the usual orthogonal or von Neumann measurement, the POVM elements need not be orthogonal, as $F_{A, x} F_{A, z} \neq 0$. But similar to von Neumann measurement, given the outcome $a$ of the POVM $a=x, y$ or $z$, the state $|\psi\rangle$ undergoing the measurement becomes $|\psi\rangle \rightarrow F_{A, a}|\psi\rangle$ [1]. It can be verified that these POVM elements satisfy the conservation of probability, i.e., $\sum_{\nu \in\{x, y, z\}} F_{A, \nu}^{\dagger} F_{A, \nu}=I_{S=3 / 2}$, where $I_{S=3 / 2}$ is the identity operator for the spin-3/2 Hilbert space. Physically, $F_{A, \nu}=\left(S_{A, \nu}^{2}-1 / 4\right) / \sqrt{6}$ is proportional to a projector onto the two-dimensional subspace spanned by the two eigenstates of the $\nu$-component spin operator with eigenvalues $S_{\nu}= \pm 3 / 2$. From the viewpoint of three virtual qubits, the POVM elements can be written as

$$
\begin{aligned}
& \tilde{F}_{A, z}=\sqrt{\frac{2}{3}}(|000\rangle\langle 000|+| 111\rangle\langle 111|), \\
& \tilde{F}_{A, x}=\sqrt{\frac{2}{3}}(|+++\rangle\langle+++|+|---\rangle\langle---|), \\
& \tilde{F}_{A, y}=\sqrt{\frac{2}{3}}(|i, i, i\rangle\langle i, i, i|+|-i,-i,-i\rangle\langle-i,-i,-i|),
\end{aligned}
$$

where $|0 / 1\rangle,| \pm\rangle \equiv(|0\rangle \pm|1\rangle) / \sqrt{2}$ and $| \pm i\rangle \equiv(|0\rangle \pm$ $i|1\rangle) / \sqrt{2}$ are eigenstates of Pauli operators $\sigma_{z}, \sigma_{x}$ and $\sigma_{y}$, respectively. Note for convenience we shall also use $|0 / 1\rangle_{a}$ (with $a=x, y$, or $z$ ) to denote eigenstates of $\sigma_{a}$, i.e., $\sigma_{a}|0 / 1\rangle_{a}= \pm|0 / 1\rangle_{a}$. We note that in this notation, $\tilde{F}$ 's can be conveniently written as

$$
\tilde{F}_{A, a}=\sqrt{\frac{2}{3}}\left(|000\rangle_{a}\langle 000|+| 111\rangle_{a}\langle 111|\right) .
$$

One advantage of using the virtual-qubit representation is that the stabilizer formalism [19] can be employed and insight about the post-POVM state can thus be gained. The above POVM elements in the second form obey the relation $\sum_{\nu \in\{x, y, z\}} \tilde{F}_{A, \nu}^{\dagger} \tilde{F}_{A, \nu}=\Pi_{S, A}$, i.e., project onto the symmetric subspace, as required. The outcome of the POVM at any site $A$ is random, which can be $x, y$ or $z$, and it can be correlated with the outcomes at other sites due to the entanglement in the AKLT state [17.

One important consequence after the POVM on a spin$3 / 2 A$ is that, even though it has four levels, its state after the POVM is restricted to the two-dimensional Hilbert subspace, spanned by $|+3 / 2\rangle$ and $|-3 / 2\rangle$, or in terms of the three virtual qubits by $|000\rangle$ and $|111\rangle$ with the quantization axis given by the POVM outcome. Thus, after the POVM, one can treat each $A$ site as the carrier of one qubit.

\section{A. Strategy}

Given the outcomes $\left\{a_{v}\right\}$ of POVMs at all center sites $\{v\}$, we know the post-POVM state is given by

$$
\begin{aligned}
\left|\psi^{\prime}\right\rangle & \sim \otimes_{v}^{\otimes} \tilde{F}_{v, a_{v}}\left|\psi_{\mathrm{AKLT}}\right\rangle \\
& \sim \otimes_{v}^{\otimes} \tilde{F}_{v, a_{v}} \underset{\text { edge } e}{\otimes}|\phi\rangle_{e}
\end{aligned}
$$

where we have used the virtual-qubit version of $F$ 's and in going from the first line to the second, we have used the fact that $\tilde{F}$ 's projects into the symmetric subspace of three qubits as well and thus there is no need to keep the projectors $\Pi_{S, v}$ 's. To understand what the post-POVM state is, we then employ the stabilizer formalism [19] and try to find its stabilizer operators. For a singlet state $|\phi\rangle_{12}=(|01\rangle-|10\rangle) / \sqrt{2}$ of two qubits 1 and 2 , it is easy to see that there are stabilizer operators [20] $-\sigma_{a}^{[1]} \otimes \sigma_{a}^{[2]}$ with $a=x, y$, or $z$, namely,

$$
-\sigma_{x} \otimes \sigma_{x}|\phi\rangle=-\sigma_{y} \otimes \sigma_{y}|\phi\rangle=-\sigma_{z} \otimes \sigma_{z}|\phi\rangle=|\phi\rangle .
$$


(a)

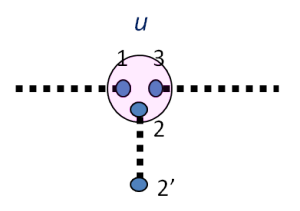

(c)

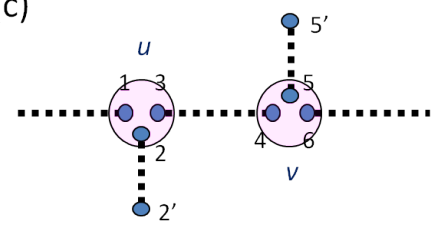

FIG. 3: (Color online) Encoding of a qubit. (a) The encoding of a qubit state for two particles: center spin-3/2u and the connected spin- $1 / 22^{\prime}$ after a POVM on $u$ giving the outcome $a_{u}$. (b) This illustrates the case where the spin- $3 / 2$ particle is located at either end, and thus it has two neighboring qubits: 0 and $2^{\prime}$. (c) When the POVM's on two consecutive sites of center spin-3/2 particles give the same outcome, i.e., $a_{u}=a_{v}$, the qubit is encoded by the two blocks, consisting two spin$3 / 2$ 's and two spin-1/2's.

The key ingredient of the stabilizer formalism applied to this example is that given two of the above commuting and independent operators, e.g., $-\sigma_{x} \otimes \sigma_{x}$ and $-\sigma_{z} \otimes \sigma_{z}$, the state $|\phi\rangle$ is uniquely determined. If operators such as the above commute with all $\tilde{F}$ 's, they will remain the stabilizer operators for the post-POVM state.

Another type of stabilizer operators arises from the POVMs. For example, using the form given in Eq. (7), we see that there are operators (two of them being independent) that satisfy

$\sigma_{a}^{[1]} \otimes \sigma_{a}^{[2]} \tilde{F}_{A, a}=\sigma_{a}^{[1]} \otimes \sigma_{a}^{[3]} \tilde{F}_{A, a}=\sigma_{a}^{[2]} \otimes \sigma_{a}^{[3]} \tilde{F}_{A, a}=\tilde{F}_{A, a}$.

Hence, these are stabilizer operators of the post-POVM state.

A third type of stabilizer operators that we shall identify below give rise to the graph-state stabilizer operators. These emerge from POVMs across a few sites. Once we identify all stabilizer operators, the state is uniquely determined if the number of independent stabilizer operators equals that of spins.

\section{B. Encoding}

Suppose the POVM on one of the center spin- $3 / 2 u$ results in $a_{u} \in\{x, y, z\}$; see Fig. 33. As seen from Eq. (7) or the stabilizer operators in Eq. 10), the three virtual qubits at $u$ are projected to the subspace spanned by $|000\rangle$ and $|111\rangle$, with $|0 / 1\rangle$ being the eigenstates of $\sigma_{a_{u}}$ (and the quantization axis being $a_{u}$ ). Furthermore, from

\begin{tabular}{|c|c|c|c|}
\hline $\begin{array}{l}\text { POVM } \\
\text { outcome }\end{array}$ & z & $x$ & $y$ \\
\hline $\begin{array}{l}\text { Stabilizer } \\
\text { generators }\end{array}$ & $\begin{array}{c}\sigma_{z}^{[1]} \sigma_{z}^{[2]}, \sigma_{z}^{[1]} \sigma_{z}^{[3]} \\
\text { and }-\sigma_{z}^{[2]} \sigma_{z}^{\left[2^{\prime}\right]}\end{array}$ & $\begin{array}{c}\sigma_{x}^{[1]} \sigma_{x}^{[2]}, \sigma_{x}^{[1]} \sigma_{x}^{[3]} \\
\text { and }-\sigma_{x}^{[2]} \sigma_{x}^{\left[2^{\prime}\right]}\end{array}$ & $\begin{array}{c}\sigma_{y}^{[1]} \sigma_{y}^{[2]}, \sigma_{y}^{[1]} \sigma_{y}^{[3]} \\
\text { and }-\sigma_{y}^{[2]} \sigma_{y}^{\left[2^{\prime}\right]}\end{array}$ \\
\hline $\begin{array}{l}\text { Logical } \\
\text { Pauli } X\end{array}$ & $\sigma_{x}^{[1]} \sigma_{x}^{[2]} \sigma_{x}^{[3]} \sigma_{x}^{\left[2^{\prime}\right]}$ & $\sigma_{z}^{[1]} \sigma_{z}^{[2]} \sigma_{z}^{[3]} \sigma_{z}^{\left[2^{\prime}\right]}$ & $\sigma_{z}^{[1]} \sigma_{z}^{[2]} \sigma_{z}^{[3]} \sigma_{z}^{\left[2^{\prime}\right]}$ \\
\hline $\begin{array}{l}\text { Logical } \\
\text { Pauli } Z\end{array}$ & $\begin{array}{c}\sigma_{z}^{[1]}, \sigma_{z}^{[2]}, \\
\sigma_{z}^{[3]} \text { or }-\sigma_{z}^{\left[2^{\prime}\right]}\end{array}$ & $\begin{array}{c}\sigma_{x}^{[1]}, \sigma_{x}^{[2]}, \\
\sigma_{x}^{[3]} \text { or }-\sigma_{x}^{\left[\left[^{\prime}\right]\right.}\end{array}$ & $\begin{array}{c}\sigma_{y}^{[1]}, \sigma_{y}^{[2]} \\
\sigma_{y}^{[3]} \text { or }-\sigma_{y}^{\left[2^{\prime}\right]}\end{array}$ \\
\hline
\end{tabular}

TABLE I: Table for local encoding. Please refer to Fig. 3 a for the qubit labeling $\left\{1,2,3,2^{\prime}\right\}$ at a site. The stabilizer generators determine the effective two levels, e.g., in the case of $z$-outcome: $|(000) 1\rangle$ and $|(111) 0\rangle$, where the qubits are listed in the order of labeling $\{1,2,3,2\}$. The logical Pauli $X$ induces transition between the two, i.e., $X \sim|(000) 1\rangle\langle(111) 0|+$ $|(111) 0\rangle\langle(000) 1|$. The logical Pauli $Z$ operators can differ by a sign due to the convention, e.g., one chooses $Z=\sigma_{z}^{1}$ and thus $Z|(000) 1\rangle=|(000) 1\rangle$ and $Z|(111) 0\rangle=-|(111) 0\rangle$. If two consecutive sites share the same POVM outcome, e.g., $z$, then the encoding extends to two sites of total 8 qubits (6 virtual ones). If the site is at the end, as in Fig. 3 3 , a corresponding table of encoding using five qubits can be similarly constructed.

the discussions above, there is a stabilizer operator for the post-POVM state, as $-\sigma_{a_{u}}^{[2]} \otimes \sigma_{a_{u}}^{\left[2^{\prime}\right]}$ commutes with $\tilde{F}_{u, a_{u}}$, where 2 denotes the virtual spin- $1 / 2$ on site $u$ that connects $2^{\prime}$. Thus, the only two possibilities of the joint state of $u$ and the connected qubit $2^{\prime}$ can be only $\left|(000)_{u} 1_{2^{\prime}}\right\rangle$ and $\left|(111)_{u} 0_{2^{\prime}}\right\rangle$, as they satisfy

$$
\begin{aligned}
& -\sigma_{a_{u}}^{[2]} \otimes \sigma_{a_{u}}^{\left[2^{\prime}\right]}\left|(000)_{u} 1_{2^{\prime}}\right\rangle=\left|(000)_{u} 1_{2^{\prime}}\right\rangle, \\
& -\sigma_{a_{u}}^{[2]} \otimes \sigma_{a_{u}}^{\left[2^{\prime}\right]}\left|(111)_{u} 0_{2^{\prime}}\right\rangle=\left|(111)_{u} 0_{2^{\prime}}\right\rangle .
\end{aligned}
$$

This means that the two physical spins $u$ and $2^{\prime}$ constitute the encoding of an effective qubit.

Now that we have identified the encoding of a qubit, we would like to seek the encoding of Pauli X and Z operators. Let us suppose $a_{u}=z$ and thus the two states $|\overline{0}\rangle \equiv\left|(000)_{u} 1_{2^{\prime}}\right\rangle$ and $|\overline{1}\rangle \equiv\left|(111)_{u} 0_{2^{\prime}}\right\rangle$ (with the basis $|0 / 1\rangle$ such that $\left.\sigma_{z}|0 / 1\rangle= \pm|0 / 1\rangle\right)$ constitute the basis states of a logical qubit. There are four equivalent choices of Pauli $Z$ : (1) $\sigma_{z}^{[1]},(2) \sigma_{z}^{[2]},(3) \sigma_{z}^{[3]}$ and (4) $-\sigma_{z}^{\left[2^{\prime}\right]}$, as these satisfy that $Z|\overline{0}\rangle=|\overline{0}\rangle$ and $Z|\overline{1}\rangle=|\overline{1}\rangle$. The four choices are equivalent, as any two of them are connected by a stabilizer operator, e.g., $\sigma_{z}^{[2]}=\sigma_{z}^{[1]}\left(\sigma_{z}^{[1]} \otimes \sigma_{z}^{[2]}\right)$. To obtain the effective Pauli X, which has action that takes $|\overline{0}\rangle$ to $|\overline{1}\rangle$ and vice versa, we can check the following combination works,

$$
X \equiv \sigma_{x}^{[1]} \otimes \sigma_{x}^{[2]} \otimes \sigma_{x}^{[3]} \otimes \sigma_{x}^{\left[2^{\prime}\right]} .
$$

On the other, when $a_{u}=x$, the two basis states are $|\overline{0}\rangle \equiv\left|(+++)_{u}(-)_{2^{\prime}}\right\rangle$ and $|\overline{1}\rangle \equiv\left|(---)_{u}(+)_{2^{\prime}}\right\rangle$, where $\sigma_{x}| \pm\rangle= \pm| \pm\rangle$. The four equivalent choices of effective Pauli $Z$ are: (1) $\sigma_{x}^{[1]},(2) \sigma_{x}^{[2]}$, (3) $\sigma_{x}^{[3]}$ and (4) $-\sigma_{x}^{\left[2^{\prime}\right]}$, as these satisfy that $Z|\overline{0}\rangle=|\overline{0}\rangle$ and $Z|\overline{1}\rangle=|\overline{1}\rangle$. The effective Pauli X can be chosen to be

$$
X \equiv \sigma_{z}^{[1]} \otimes \sigma_{z}^{[2]} \otimes \sigma_{z}^{[3]} \otimes \sigma_{z}^{\left[2^{\prime}\right]},
$$


as $X|\overline{0}\rangle=|\overline{1}\rangle$ and $X|\overline{1}\rangle=|\overline{0}\rangle$. Effectively, the POVM outcome $a_{u}$ indicates the new quantization axis for the effective qubit. For a summary of stabilizer generators, effective Pauli $X$ and $Z$ operators, please refer to Table $[$. which also includes the case of $a_{u}=y$ outcome.

As exemplified in Fig. 3b, if the center spin-3/2 is located at either end, it has two virtual spin- $1 / 2$ 's connected to it. The three sites form the basis of the qubit encoding: $\left|(000)_{u} 1_{0} 1_{2^{\prime}}\right\rangle$ and $\left|(111)_{u} 0_{0} 0_{2^{\prime}}\right\rangle$.

Now suppose the POVM's on several consecutive sites of center spin- $3 / 2$ result in a same outcome; see the example in Fig. 35 for two sites with $a=a_{u}=a_{v}$. Those spin-3/2's and the connected virtual spin-1/2's together form the encoding of a logical qubit. This is due to additionally that there is a stabilizer operator $-\sigma_{a}^{[3]} \otimes \sigma_{a}^{[4]}$ for the virtual qubits on the two of the neighboring center sites $u$ and $v$ with the same POVM outcome $a$ and that $-\sigma_{a}^{[3]} \otimes \sigma_{a}^{[4]}$ commutes with both $\tilde{F}_{u, a}$ and $\tilde{F}_{v, a}$. Therefore, the two blocks that consists of two spin-3/2's $(u$ and $v)$ and two spin-1/2's $\left(2^{\prime}\right.$ and $\left.5^{\prime}\right)$ altogether only encode one qubit with two basis states being $\left|(000)_{u} 1_{2^{\prime}}(111)_{v} 0_{5^{\prime}}\right\rangle$ and $\left|(111)_{u} 0_{2^{\prime}}(000)_{v} 1_{5^{\prime}}\right\rangle$. This result generalizes to any number of consecutive center sites having the same POVM outcome [14. When all spin-3/2 sites along a chain has been performed of POVM, there is a sequence of POVM outcomes, e.g., $x x y z x z z z y \cdots$. Consecutive sites of the same outcome (e.g., $x x$ and $z z z$ ) are grouped into what we call a domain.

We remark that by single-spin measurements, the encoding of a logical qubit by a domain can be reduced to a single site. For example, if one measures the spin at site $v$ in the basis $| \pm\rangle \equiv(|0\rangle \pm|1\rangle) / \sqrt{2}$, a state $\alpha\left|(000)_{u} 1_{v}\right\rangle+$ $\beta\left|(111)_{u} 0_{v}\right\rangle$ will be reduced to $\alpha\left|(000)_{u}\right\rangle \pm \beta\left|(111)_{u}\right\rangle$, depending on the outcome of the measurement. Similarly, if one measures the spin at site $u$ in the basis $(|000\rangle \pm|111\rangle) / \sqrt{2}$, which is, in terms of spin-3/2 language, $(|+3 / 2\rangle \pm|-3 / 2\rangle) / \sqrt{2}$, the post-measurement state becomes $\alpha\left|0_{v}\right\rangle \pm \beta\left|1_{v}\right\rangle$. Thus, an encoded cluster state can thus be reduced by local measurement to a cluster state, where each site is effectively a qubit. For simplicity, from now on we shall focus on cases where no neighboring sites sharing the same outcome for illustration.

\section{Cluster-state stabilizer}

To show that the post-POVM CMDB state is an (encoded) cluster state, let us take the example shown in Fig. 4. Let us labels the three center sites in blocks $U$, $V$ and $W$ by the lower-case symbols $u, v$ and $w$ respectively. Suppose the POVM outcomes on these sites are $a_{u}=x, a_{v}=z$, and $a_{w}=x$, respectively. First note that $-\sigma_{x}^{[3]} \sigma_{x}^{[4]}$ commutes with $\tilde{F}_{u, x}$ and $-\sigma_{x}^{[6]} \sigma_{x}^{[7]}$ commutes with $\tilde{F}_{u, x}$. Note also that $-\sigma_{x}^{[5]} \sigma_{x}^{\left[5^{\prime}\right]}$ is a stabilizer operator of the singlet between 5 and $5^{\prime}$, but it does not commute with $\tilde{F}_{v, z}$. However, if we multiply all the above

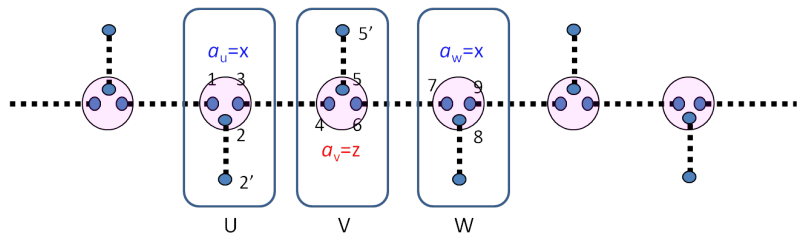

FIG. 4: (Color online) Cluster-state stabilizer operator. The example shows POVM outcomes on the center sites $u, v$, and $w$ are $a_{u}=x, a_{v}=z$, and $a_{w}=x$, respectively. It can be shown that for the post-POVM state there is a stabilizer operator defined on the three blocks $U, V$, and $W: \bar{Z}_{U} \bar{X}_{V} \bar{Z}_{W}$, up to a possible sign.

operators, we obtain

$$
K_{V} \equiv-\sigma_{x}^{[3]} \sigma_{x}^{[4]} \sigma_{x}^{[5]} \sigma_{x}^{\left[5^{\prime}\right]} \sigma_{x}^{[6]} \sigma_{x}^{[7]}
$$

Because $K_{V}$ commutes with $\tilde{F}_{v, z}$, due to the identity

$$
\begin{aligned}
& \sigma_{x} \otimes \sigma_{x} \otimes \sigma_{x}(|000\rangle\langle 000|+| 111\rangle\langle 111|) \\
= & (|111\rangle\langle 000|+| 000\rangle\langle 111|) \\
= & (|000\rangle\langle 000|+| 111\rangle\langle 111|) \sigma_{x} \otimes \sigma_{x} \otimes \sigma_{x},
\end{aligned}
$$

it is a stabilizer operator of the post-measurement state. In terms of logical Pauli operators $Z_{U} \equiv \sigma_{x}^{[3]}, Z_{W} \equiv \sigma_{x}^{[7]}$, $X_{V} \equiv \sigma_{x}^{[4]} \sigma_{x}^{[5]} \sigma_{x}^{[6]} \sigma_{x}^{\left[5^{\prime}\right]}$ (see the examples given in the previous section for the effective Pauli operators), we arrive at the stabilizer operator $K_{V}=-Z_{U} X_{V} Z_{W}$. This is (up to a sign) the stabilizer operator defining a linear cluster state [4]. We note that for completeness of the qubit encoding for blocks $U, V$ and $W$, the corresponding conjugate operators $Z$ or $X$ can be chosen as follows: $X_{U} \equiv \sigma_{z}^{[3]}, X_{W} \equiv \sigma_{z}^{[7]}$ and $Z_{V} \equiv \sigma_{z}^{[4]}$ and that they satisfy the required anticommutation with their conjugate operators.

Note that the choice of three consecutive sites (or domains) is arbitrary. Furthermore, if the above example is changed to a four-site problem, e.g., with the POVM outcomes being $x z z x$, i.e., with middle two sites having the same $z$ outcome. These two sites form a single logical qubit, as discussed earlier in Sec. IVB and the encoded Pauli $X$ operators will span across the two sites. The above example can be straightforwardly generalized to a general proof that the state after the POVM is, under local unitary transformation, equivalent to an encoded cluster state, similar to the case of 2D AKLT state on the honeycomb lattice [14]. (The encoding of logical qubits can be reduced to single sites by suitable local measurements, as remarked earlier.) As a further illustration, let us consider another example of three sites in Fig. 4 but with $a_{u}=x, a_{v}=z$, and $a_{w}=y$, i.e., the last site has a different outcome $a_{w}=y$ than the above example. Because of this, one now considers $-\sigma_{y}^{[6]} \sigma_{y}^{[7]}$ instead of $-\sigma_{x}^{[6]} \sigma_{x}^{[7]}$ and can show that the follow operator is a stabilizer generator:

$$
K_{V} \equiv-\sigma_{x}^{[3]} \sigma_{x}^{[4]} \sigma_{x}^{[5]} \sigma_{x}^{\left[5^{\prime}\right]} \sigma_{y}^{[6]} \sigma_{y}^{[7]} .
$$


Now, one can choose $Z_{W} \equiv \sigma_{y}^{[7]}, Z_{U} \equiv \sigma_{x}^{[3]}, Z_{W} \equiv \sigma_{y}^{[7]}$, $X_{V} \equiv \sigma_{x}^{[4]} \sigma_{x}^{[5]} \sigma_{x}^{[6]} \sigma_{x}^{\left[5^{\prime}\right]}$, and $Z_{V} \equiv \sigma_{z}^{[6]}$ (see Table I) and obtain

$$
K_{V}=-Z_{U}\left(X_{V} i Z_{V}\right) Z_{W}=-Z_{U} Y_{V} Z_{W} .
$$

Although the stabilizer operator $K_{V}$ is not of the canonical form of the cluster-state stabilizer $Z_{U} X_{V} Z_{W}$, they are related by local unitary transformation that leaves $Z_{V}$ invariant.

The hidden cluster state in the ground state of the AKLT quasichain is hence revealed by the POVM (5) or equivalently (6). The original 1D spin-1 AKLT state has previously been shown to be locally converted to a $1 \mathrm{D}$ cluster state by a similar spin-1 POVM [14 and by a different alternating POVM [21].

\section{QUANTUM COMPUTATIONAL UNIVERSALITY OF THE 2D CAI-MIYAKE-DÜR-BRIEGEL STATE}

After understanding that the quasichain AKLT state can be converted to an (encoded) 1D cluster state, arbitrary single-qubit rotations can be implemented along every quasichain in the usual one-way computation linear cluster states 2. What remains to be seen for the quantum computational universality is whether entangling gates such as control-NOT or control-phase can be implemented between the two logical qubits on two neighboring quasichains. The answer is affirmative. The two spin- $1 / 2 b$ particles on two neighboring chains, which are the two virtual qubits of the merged $B$-type spin- $3 / 2$ particle, are used as either (1) a disconnecter or (2) a connecter so that either in case (1) the two neighboring logical qubits evolve independently (without being acted upon by an entangling gate) or case (2) an entangling gate acts on them. Either functionality is achieved by measurement on a $B$-site particle or equivalently by the joint measurement of two $b$ particles; see Fig. 5 .

\section{A. Connecting/disconnecting neighboring logical qubits}

In the case of a disconnecter, the two virtual spin- $1 / 2$ particles (of the single spin $B$ ) are each applied an effective Pauli $\mathrm{X}$ gate $X=|0\rangle_{a}\langle 1|+| 1\rangle_{a}\langle 0|[22$ before being measured in the basis of respective $| \pm\rangle_{a}$, where the axis label $a$ can be either $x, y$, or $z$ (depending on the POVM outcome on the neighboring spin-3/2 particle) and $| \pm\rangle_{a} \equiv\left(|0\rangle_{a} \pm|1\rangle_{a}\right) / \sqrt{2}$ with $\sigma_{a}|0 / 1\rangle_{a}= \pm|0 / 1\rangle_{a} ;$ see Fig. 5. Suppose the outcome of the measurement on the spin- $1 / 2$ connected to the spin-3/2 $u$ is labeled by $(-1)^{m_{1}}$ with $m_{1}=0 / 1$, and similarly for the one connected to $v:(-1)^{m_{2}}$. (Together, $m_{1}$ and $m_{2}$ characterize the measurement outcome of the merged spin-3/2 B.) Then the effect of measuring the spin $B$ on neighboring $u$ and $v$

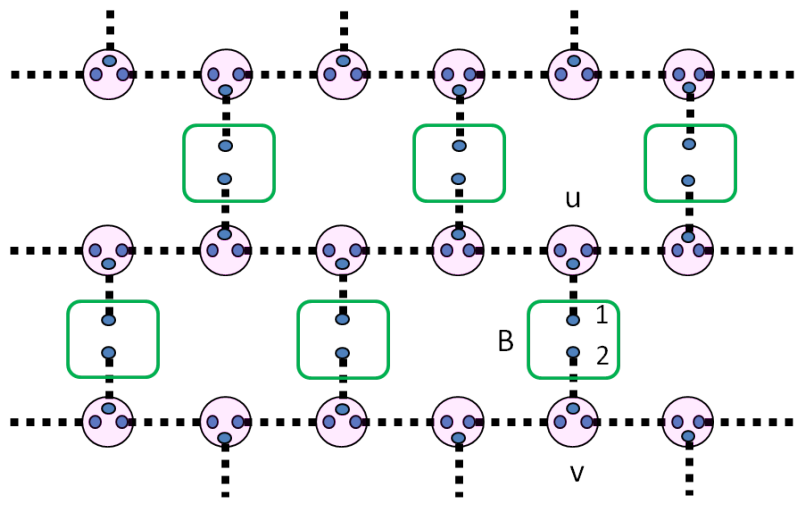

FIG. 5: (Color online) 2D structure. The two spin-1/2 $b$ particles (labeled 1 and 2) on two neighboring chains, are the two virtual qubits of the merged $B$-type spin-3/2 particle. By measuring the $B$ spin, two spins $u$ and $v$ will (i) evolves independently or (ii) be acted upon by a control-phase gate.

is that single-qubit gates $Z_{u}^{m_{1}} \otimes Z_{v}^{m_{2}}$ have been applied to $u$ and $v$, where $Z_{u}=|3 / 2\rangle_{a_{u}}\langle 3 / 2|-|-3 / 2\rangle_{a_{u}}\langle-3 / 2|$ and similarly for $Z_{v}$. Note that in the three-virtual-qubit picture, $|+3 / 2\rangle_{a_{u}}=|000\rangle_{a_{u}}$ and $|-3 / 2\rangle_{a_{u}}=|111\rangle_{a_{u}}$. Furthermore, the measurement on 1 and 2 corresponds to a local measurement on the spin- $3 / 2 B$. The logical qubits on neighboring chains evolve independently, i.e., no entangling gate has been applied to $u$ and $v$.

In the case of a connecter, the two spin- $1 / 2$ particles are first applied effective Pauli $\mathrm{X}$ gates: $X=$ $|0\rangle_{a}\langle 1|+| 1\rangle_{a}\langle 0|$ and then a control-phase (CP) gate: $\mathrm{CP}_{12}=|0\rangle_{a_{u}}\left\langle\left. 0\right|^{[1]} \otimes \mathbb{1}^{[2]}+\mid 1\right\rangle_{a_{u}}\left\langle\left. 1\right|^{[1]} \otimes Z_{a_{v}}^{[2]}\right.$, where $Z_{a_{v}}=$ $|0\rangle_{a_{v}}\langle 0|-| 1\rangle_{a_{v}}\langle 1|$. These operations correspond a singlespin unitary transformation on the merged $B$ spin. Then, a measurement in their respective $| \pm\rangle_{a}$ basis is made as in the previous case, and the effect is that an entangling gate has acted on the two associated spin- $3 / 2$ particles $u$ and $v$ (which are effectively two qubits), up to Pauli gates. Using the measurement labels $m_{1}$ and $m_{2}$, the entangling gate that has been applied is $Z_{u}^{m_{1}} \otimes Z_{v}^{m_{2}} \mathrm{CP}_{u v}$, where

$\mathrm{CP}_{u v}=\left|\frac{3}{2}\right\rangle_{a_{u}}\left\langle\left.\frac{3}{2}\right|^{[u]} \otimes I_{a_{v}}^{[v]}+\mid-\frac{3}{2}\right\rangle_{a_{u}}\left\langle-\left.\frac{3}{2}\right|^{[u]} \otimes Z_{a_{v}}^{[v]}\right.$,

where $I_{a_{v}}^{[v]}=|3 / 2\rangle_{a_{v}}\langle 3 / 2|+|-3 / 2\rangle_{a_{v}}\langle-3 / 2|$ is the effective qubit identity operator and $Z_{a_{v}}=|3 / 2\rangle_{a_{v}}\langle 3 / 2|-$ $|-3 / 2\rangle_{a_{v}}\langle-3 / 2|$ is the effective Pauli $\mathrm{Z}$ operator. The logical qubits on neighboring chains have thus undergone a control-phase gate up to Pauli Z's.

We remark that the approach of the joint measurement on the two virtual qubits has also been used recently in Refs. [13] and 23] and was employed earlier in Ref. 24]. In summary, an entangling gate can indeed be applied on two logical qubits residing on the two neighboring quasichains. Thus, we see that CMDB state is a universal resource for MBQC. 
(a)

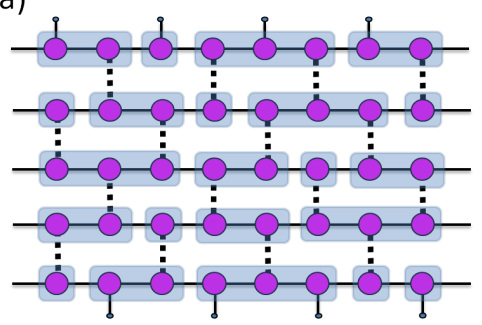

(b)

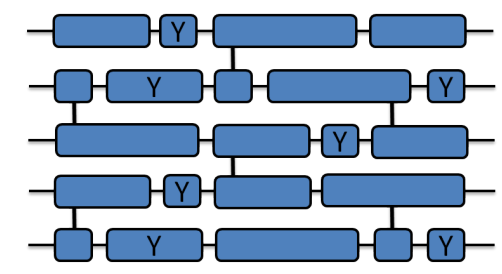

(c)

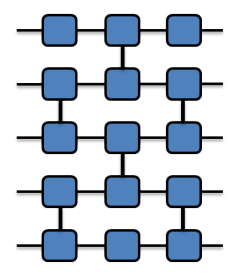

FIG. 6: (Color online) Converting the Cai-Miyake-DürBriegel state to a $2 \mathrm{D}$ cluster state. (a) The shaded boxes enclose sites that share the same POVM outcomes on $A$ sites. The $B$ sites are not shown explicitly and only dashed lines are shown. (b) The solid vertical lines denote the locations where CP gates are applied. Boxes labeled with ' $\mathrm{Y}$ ' indicate subsequent logical Pauli Y measurements to be made. (c) After the Pauli Y measurements, the left and right neighboring logical qubits, represented by boxes, are linked. This gives rise to a $2 \mathrm{D}$ cluster state.

\section{B. Converting to a $2 \mathrm{D}$ cluster state}

The whole system can be further converted to a $2 \mathrm{D}$ cluster state on a honeycomb lattice. First, a structure which is topologically equivalent to a honeycomb (or brick-wall) lattice can be identified. Second, by suitable disconnecting or connecting measurements on $B$ sites and then Pauli measurements on $A$ sites, the structure can be reduced to a honeycomb lattice. Shown in Fig. 6 is the example of five quasichains. In Fig. 6a, the shaded boxes enclose sites that share the same POVM outcomes on $A$ sites. The $B$ sites are not shown explicitly and only vertical dashed lines are used to indicate the possible connecter/disconnecter roles by $B$ spins. In Fig. 6p, the solid vertical lines denote the choice of a connecter (and the remaining dashed vertical lines in Fig. 6a denote disconnecters) and hence a control-phase gate acts on two neighboring $A$ sites connected by solid vertical lines. The dangling spin-1/2's sites are omitted. Those boxes with a ' $\mathrm{Y}$ ' represent a subsequent Pauli-Y mea-

surement to remove the qubit on the sites and to link its two neighboring blocks, resulting in a graph state that has the brickwall structure, as shown in Fig. 6r. This illustrates and generalizes to a proof that a $2 \mathrm{D}$ cluster state can be distilled from the CMDB state.

\section{CONCLUDING REMARKS}

In summary, we provide an alternative understanding of the quantum computational universality of the $2 \mathrm{D}$ state constructed by Cai, Miyake, Dür and Briegel [13]. A crucial step is the preprocessing given by the POVM (5) on the central $A$ sites of all chains, which converts each chain to an encoded 1D cluster state and thus arbitrary single-qubit rotation can be implemented. Entangling gates, such as control-phase and control-NOT gates, between neighboring two logical qubits can be implemented by measuring the linking spin-3/2 $B$ sites. The whole system can also be further converted to a $2 \mathrm{D}$ cluster state.

The quantum computation in the correlation space proposed by Gross and Eisert [8] was initially regarded as a scheme that belongs to the so-called CCuniversality [25], where the quantum computer takes classical input and delivers classical output. Many of the resource states constructed in Ref. [8] were later shown to be locally convertible to cluster states [21, 26], thus enabling the so-called CQ-universality, in which a quantum computer takes a classical input but can deliver a quantum state as a output. Quantum computers of CQ-universality seems potentially more powerful than those of CC-universality. By showing that a 2D cluster state can be distilled by local operations, we have in turn shown that the Cai-Miyake-Dür-Briegel state enables a CQ-universal quantum computation. Whether CQ-universality class is indeed more powerful than CCuniversality class in general remains open. In view of resource states, this raises the question whether or not there exists a resource state for which measurementbased quantum computation can only be carried out in the correlation space but cannot be done in the Hilbert space of physical spins.

Acknowledgment. We thank Maarten Van den Nest for useful discussions. This work was supported by NSERC (T.-C.W. and R.R.), MITACS (T.-C.W. and R.R.), CIFAR (R.R.), the Sloan Foundation (R.R.), IARPA (R.R.) and National Research Foundation and Ministry of Education of Singapore (L.-C.K.).
[1] M. Nielsen and I. Chuang, Quantum Computation and Quantum Information (Cambridge Univ. Press, 2000).

[2] R. Raussendorf and H. J. Briegel, Phys. Rev. Lett. 86, 5188 (2001).

[3] H. J. Briegel, D. E. Browne, W. Dür, R. Raussendorf, and M. Van den Nest, Nature Phys. 5, 19 (2009).
[4] H. J. Briegel and R. Raussendorf, Phys. Rev. Lett. 86, 910 (2001).

[5] M. Hein, J. Eisert, and H.-J. Briegel, Phys. Rev. A 69, 062311 (2004).

[6] M. Van den Nest, A. Miyake, W. Dür, and H. J. Briegel, Phys. Rev. Lett. 97, 150504 (2006). 
[7] D. Gross, S. T. Flammia, and J. Eisert, Phys. Rev. Lett. 102, 190501 (2009); M. Bremner, C. Mora, and A. Winter, ibid 102, 190502 (2009).

[8] D. Gross and J. Eisert, Phys. Rev. Lett. 98, 220503 (2007); D. Gross, J. Eisert, N. Schuch, and D. PerezGarcia, Phys. Rev. A 76, 052315 (2007); D. Gross and J. Eisert, Phys. Rev. A 82, 040303(R) (2010).

[9] F. Verstraete and J. I. Cirac, Phys. Rev. A 70 060302(R) (2004).

[10] Measurement-based quantum computation with these new resource states take place in the so-called "correlation space" rather than the Hilbert space of physical qubits; see Ref. [8].

[11] S. D. Bartlett and T. Rudolph, Phys. Rev. A 74, 040302(R) (2006).

[12] X. Chen, B. Zeng, Z.-C. Gu, B. Yoshida, and I. L. Chuang, Phys. Rev. Lett. 102, 220501 (2009).

[13] J.-M. Cai, A. Miyake, W. Dür, and H. J. Briegel, Phys. Rev. A 82, 052309 (2010).

[14] T.-C. Wei, I. Affleck, and R. Raussendorf, Phys. Rev. Lett. 106, 070501 (2011); T.-C. Wei, I. Affleck, and R. Raussendorf, e-print arXiv:1009.2840

[15] A. Miyake, Ann. Phys. (Leipzig) 326, 1656 (2011).

[16] M. A. Nielsen, Rep. Math. Phys. 57, 147 (2005).

[17] I. Affleck, T. Kennedy, E. H. Lieb, and H. Tasaki, Phys. Rev. Lett. 59, 799 (1987); I. Affleck, T. Kennedy, E. H. Lieb, and H. Tasaki, Comm. Math. Phys. 115, 477 (1988).

[18] G. K. Brennen and A. Miyake, Phys. Rev. Lett. 101, 010502 (2008).
[19] D. Gottesman, Stabilizer Codes and Quantum Error Correction, Ph.D. Thesis, Caltech (1997).

[20] Stabilizer operators $K_{v}$ in the stabilizer $\mathcal{S}$ are unitary operators that commute with one another. $K_{v}$ is a stabilizer operator of the state $|\psi\rangle$ if it satisfies $K_{v}|\psi\rangle=|\psi\rangle$. One important property of qubit stabilizer operators is that when the number of independent stabilizer operators equals that of the total number of qubits in $|\psi\rangle$, the state is uniquely determined. If there is one independent stabilizer less, then the state is only determined up to a subspace of an effective qubit. For details, see Ref. [19].

[21] X. Chen, R. Duan, Z. Ji, and B. Zeng, Phys. Rev. Lett. 105, 020502 (2010).

[22] The reason to apply a Pauli $\mathrm{X}$ is to flip the encoding states of the $b$ and its assocaite $A$ particles from $\left|(000)_{A} 1_{b}\right\rangle$ and $\left|(111)_{A} 0_{b}\right\rangle$ [see Eq. (11]] to $\left|(000)_{A} 0_{b}\right\rangle$ and $\left|(111)_{A} 1_{b}\right\rangle$, respectively, is for convenience of later analysis. One then investigates the effect on $A_{1}$ and $A_{2}$ due to the measurement on the associated $b_{1}$ and $b_{2}$ in the $| \pm\rangle$ basis. In the case of a connecter, an additional control-phase gate is applied to $b_{1}$ and $b_{2}$ before measurement.

[23] Y. Li, D. E. Browne, L. C. Kwek, R. Raussendorf, and T.-C. Wei, Phys. Rev. Lett. 107, 060501 (2011).

[24] D. Gottesman and I. Chuang, Nature 402, 390 (1999).

[25] M. Van den Nest, W. Dür, A. Miyake, and H. J. Briegel, New J. Phys. 9, 204 (2007).

[26] J.-M. Cai, W. Dür, M. Van den Nest, A. Miyake, and H. J. Briegel, Phys. Rev. Lett. 103, 050503 (2009). 